\title{
Editorial
}

\section{Big data analysis techniques for intelligent systems}

\author{
Ahmed Farouk $^{\mathrm{a}, *}$ and Dou Zhen ${ }^{\mathrm{b}}$ \\ ${ }^{\mathrm{a}}$ Wilfrid Laurier University, Canada \\ ${ }^{\mathrm{b}}$ Dean of School of Computer Science, Harbin Engineering University, China
}

Guest editors: Ahmed Farouk, Dou Zhen

Keywords: Big data analysis, scientific computing, data-driven service management, data processing for intelligent systems

Recently, Internet of Things (IoT), Cloud, Big Data and AI gained a great attention as the most research topics. They cover not only information and communication technology, but also all kinds of intelligent systems in our society, including business, finance, industry, manufacture, management, and environment. As a result of the extensive use of these new technologies, a huge volume of data in different formats are generated much faster than before. Big data, characterized by high volume, variety and velocity, increasingly drives decision making and is changing the landscape of intelligent systems, from governments, organizations, communities to individual decision making. Cognitive big data analytics that discovers insights from pieces of evidence has a high demand for computing efficiency, knowledge discovery, problem-solving, and event prediction/prescription. It also poses great challenges in terms of data, process, analytical modelling and management for organizations to turn big data into big insight. Due to the increase in data size, complexity, and formats, it is no longer practical to anticipate and model all possible interactions and data processing in big data-based intelligent systems using the

\footnotetext{
*Corresponding author. Ahmed Farouk, Wilfrid Laurier University, Canada. E-mail: afarouk@wlu.ca.
}

traditional data processing models. The emergence of new engineering research areas is clear evidence of the urgent needs of new demands and requirements of complex systems to be more intelligent. Besides their efficiency in quantitative analysis, big data analytics algorithms have successfully treated qualitative issues including knowledge-based decision support systems. Using these algorithms for integrating big data analytics with intelligent systems is an essential step toward achieving system goals.

The theme of this special issue is to provide an in-depth analysis both theoretically and analytically of the current advances in the field of cognitive big data analytics for intelligent systems. We are soliciting original contributions in both theoretical studies and state-of-the-art practical applications. We through call for paper through some conference and research community, we collected more than 246 papers. Based on the peer-review comments, we carefully selected 50 papers for this special issue. Now we will introduce the accepted 50 papers briefly.

The Internet of Things (IoT) promises to reshape entire industries. The business value can be profound-ranging from digitizing an organization's internal operations and customer experience to unlocking disruptive new digital products and business models. There are fifteen papers focused on 
IoT and its application technologies. The establishment of physical education courses in colleges plays a positive role in promoting the personal development of students. However, as the teaching time of the physical education courses is limited, it has become an urgent problem to improve the students' professional skills in the limited time of teaching. How to solve the problem that Fuzzy measure can't be fused. Article [32] proposed a new algorithm to solve this problem, the experimental results show the proposed algorithm can archive a great performance improvement. Article [33] discussed the adaptation of IoT technology to the needs of modern enterprise human resource management, enriching and optimizing the training mode of innovative talents in science and engineering colleges. Article [34] proposed an optimization and update scheme from algorithm flow and evaluation model, and then established an effective identification model effectively by evaluating the data connection status of financial model. Based on computer and image processing technology. Article [35] proposed an intelligent transportation video tracking technology. Article [36] proposed an automatic password update technology and applied this technology into the wireless network services. Article [37] constructed a new mechanism and algorithm for cross border e-commerce export logistics mode from the perspective of value chain. Article [38] studied the function and significance of the history teaching of art design in the development of network of things of business design. Article [39] studied the construction of teaching platform of drama film and article [40] constructed the teaching evaluation index system of track and field general course for physical education major in light of wireless network technology. Article [41] studied the temperature rise online detection system of complete switchgear based on branch demarcation algorithm.

There are nine papers are focused on data mining technologies and the algorithms for big data. Article [12] proposed a prefix span algorithm for intrusion detection, which can up to $2 \mathrm{X}$ speed up than the mostly used start-of-art methods. Article [13] uses data mining algorithms to analyze the effectiveness of ideological and political education. Article [14] studied the characteristic of ensemble learning and compared the performance of K-means clustering algorithm, Ada-Boost algorithm and support vector machine algorithm. Article [15] designed a set of experiments which compared some highly used algorithm in computer network educational administration management system. Then the author designed an improved algorithm to improve the system performance. Article [17] proposed a new simulation model to evaluate the security of computer network. In order to better apply traditional cultural symbols to visual communication design based on this model, article [16] proposed a new genetic based algorithm to superimpose the visual communication performance. In order to study the effect of empty hole between adjacent blast holes in the perforation process of blasting, article [1] proposed a rock blasting theory based simulation method to process the rock fragmentation, experimental results show the proposed method can greatly improve system performance. Article [2] takes advantage of the data mining technology to analyze the smeared as criteria to guide clinicians to prescribe medications for patients so as to reduce the pain of patient, improve medical quality and save limited health resources. Article [3] makes an empirical study on the relationship between quality management, organizational innovation and organizational performance, which get some useful conclusions.

Deep learning is an important role in artificial intelligent techniques, there are eleven papers are focused on deep learning technologies and applications. Article [18] proposed a new Prefix Span algorithm which without produce candidate sets in the process of mining, experimental results show the proposed algorithm efficiency is much higher than that of other classical algorithms. Based on the neural network model, article [19] proposed a new simulation method of computer network security. In order to promote the development of visual target tracking technology, article [20] uses discrete differential algorithm in computer to assist analysis. Article [21] studied the influence factors of the stability of tailings dam based on multi-source information fusion method. Article [22] improved genetic algorithm and then applied the proposed algorithm to evaluate the labor relations in human resource development. Focused on the real-time dispatching of people, article [49] applied the BP neural network technology to the real-time dispatching of intelligent people, establish the time prediction model of intelligent dispatching of people based on BP neural network. Article [50] presents a fuzzy optimization model for demand-responsive feeder transit services (DRT) that can transport an uncertain number of passengers from demand points to the rail station, the proposed model features fuzzy triangular number variables used to describe the changes in travel demand. To studies and optimizes the fault diagnosis method of comprehensive logging 
unit pressure sensor, article [4] proposed an improved particle swarm optimization RBF neural network algorithm to establish the temperature compensation model. In order to improve the classification effect of the gene expression profile with high dimension and small sample, article [5] proposed an improved rotation forest algorithm. The traditional sentiment classification methods are not fine-grained enough to fully characterize the overall evolution of sentiments and face the problem of sparse features. Aiming at these limitations, article [6] proposed a hybrid neural network model for fine-grained emotion classification and computing. Based on dynamic particle swarm optimization (DPSO) for BP neural network, article [11] studied the anti-glycation activity.

Cloud computing is another hot topic for nowadays research, we accept four papers on cloud computing in this special issue. Article [23] construct a new internet data mining model. Article [24] studied the breakthrough and innovation of UAV mission planning method based on cloud computing-based reinforcement learning algorithm. Article [25] studied the driving model of innovation of business model and applied the proposed model under cloud computing environment. Article [26] developed a new chosen model for comparative education research in colleges and universities in the age of big data.

There are sixteen papers which focused on intelligent algorithm. Because of all these sixteen papers are relevant with intelligent algorithm, so we introduce all these papers together. Article [27] constructed and analyzed the hidden Markov model for piano notes recognition algorithm. Article [28] applied graph theory into transportation linkage in logistics management and then designed a new computer aided model. Article [29] studied the inheritance and innovation mode of erhu art and then developed a new intelligent algorithm to improve the system performance. Article [30] explores the application of artificial intelligence in English teaching in middle schools by using relevant theories of curriculum theory, literature analysis and field investigation. Article [31] discussed the business English writing training strategy based on new media and exercise recommendation algorithm. Based on "The preservation of endangered ethnic minority languages" and "application of ethnic minority languages", article [42] combined quantitative and qualitative analysis and then proposed a new method which can carry out a systematic study on vowels and consonants in the Salar language. Article [43] investigates the impacts that salary variation caused by insufficient information, then evaluates the condition of information holding between the employee and the employer. Article [44] designed an measurement model and simulation model of electromagnetic flow transducer (EFT) with saddle excitation structure and then analyzed the distribution characteristics of magnetic flux density of different excitation structures. Article [45] focused on the study of constructing efficient two-dimensional wavelet synopses with maximum error bound. The authors proposed a linear-time twodimensional F-shift algorithm (TDFS), then present a general parallel framework for two-dimensional data array and generate a parallel two-dimensional F-shift algorithm (PTDFS). Article [46] aims to solve the problem of false positives and class imbalances by implementing an improved filter library framework for Cole pest identification. The specific impact of different passenger behaviors on crowd stampede risk was quantitatively analyzed through simulation. The research results of article [47] can be applied to the safety managements to help reduce the occurrence of stampede accidents on the escalator and promote the safety of escalator passengers. Machinery and equipment are widely used in modern large-scale production, while industrial large-scale production and the progress of science and technology make machinery and equipment more complex and large-scale, article [48] proposed a new fault prediction and maintenance process on the basis of operating state of the device, experimental results show the proposed method can improve the system performance. Article [7] studied the electrical output characteristics of photovoltaic cell and then proposed a mathematical model for engineering application. Article [8] established an evolutionary game model between government and consumers, and then discussed the equilibrium stability of the model system. In order to monitor the atmospheric pollutant of PM2.5, article [9] proposed a new BP neural network based prediction model for air quality. Article [10] proposed a new approach to solving bipartite matching problems with linguistic scorings.

In conclusion, this special issue would not have been possible without the help of many people. As guest editors, we would like to take this opportunity to thank the authors for their contributions and the reviewers for their invaluable comments and timely responses. We also would like to thank the JIFS Editor-in-Chief and staff for their support during the preparation and production of this special issue. 


\section{References}

[1] H.C. Li, X.T. Zhang, D. Li, L.M. Wu, W.L. Gao and H.M. Zhou, Numerical simulation of the effect of empty hole between adjacent blast holes in the perforation process of blasting, Journal of Intelligent \& Fuzzy Systems (JIFS).

[2] Y. Zhao, J.J. Le, L.F. Zhu and M. Zuo, Study on the effect of hypertensive treatment based on drug factor analysis model under the background of big data, Journal of Intelligent \& Fuzzy Systems (JIFS).

[3] F. Zhou and X.M. Gu, Fuzzy impact of quality management on organizational innovation performance, Journal of Intelligent \& Fuzzy Systems (JIFS).

[4] H.B. Liang, H.F. Chen and Y.J. Lu, Research on sensor error compensation of comprehensive logging unit based on machine learning, Journal of Intelligent \& Fuzzy Systems (JIFS).

[5] T. Chen, The classification of gene expression profiles based on improved rotation forest algorithm, Journal of Intelligent \& Fuzzy Systems (JIFS).

[6] W. Zhang, M. Wang, Y.C. Zhu, J. Wang and N. Ghei, A hybrid neural network approach for fine-grained emotion classification and computing, Journal of Intelligent \& Fuzzy Systems (JIFS).

[7] Y. Zhang, H.X. Sun and Y.J. Guo, Research on maximum power point tracking method of photovoltaic power generation, Journal of Intelligent \& Fuzzy Systems (JIFS).

[8] K. Liu, H.M. Zhou, Y.Y. Kou, J.B. Zhang, X.J. Zhou and X.T. Zhang, Simulation study on passive buildings' demand incentive based on evolutionary game, Journal of Intelligent \& Fuzzy Systems (JIFS).

[9] Y.G. Chen, J.M. An, W.N. Chiem and Y. Han, A novel prediction model of PM2.5 mass concentration based on back propagation neural network algorithm, Journal of Intelligent \& Fuzzy Systems (JIFS).

[10] Q. Yue and L.L. Zhang, Linguistic scoring bipartite matching considering stability and satisfaction degrees, Journal of Intelligent \& Fuzzy Systems (JIFS).

[11] B.F. Liu, Research on anti-glycation activity based on dynamic particle swarm optimization for BP neural network, Journal of Intelligent \& Fuzzy Systems (JIFS).

[12] R.S. Zhang, Network security of prefix span algorithm for data mining, Journal of Intelligent \& Fuzzy Systems (JIFS).

[13] S.Y. Zhang, J.F. Li and M. Jodylf, The related factors analysis of ideological and political effectiveness in self-media based on data mining, Journal of Intelligent \& Fuzzy Systems (JIFS).

[14] X.D. Lu and E.P. Gong, Intelligent clustering analysis model for mining area mineral resource prediction, Journal of Intelligent \& Fuzzy Systems (JIFS).

[15] H.X. Sun, Study on application of data mining technology in university computer network educational administration management system, Journal of Intelligent \& Fuzzy Systems (JIFS).

[16] L. Zhou and J.Y. Cho, A new method of design based on genetic algorithm analysis of the application of traditional cultural symbols in visual communication design, Journal of Intelligent \& Fuzzy Systems (JIFS).

[17] Y. Tang, Computer network security evaluation simulation model based on neural network, Journal of Intelligent \& Fuzzy Systems (JIFS).

[18] Q.Z. Li, Z.Q. Wang and Y. Wang, Numerical simulation study of gas explosion in confined space based on deep learning algorithm, Journal of Intelligent \& Fuzzy Systems (JIFS).

[19] H.B. Wang and H. Li, Research on theoretical analysis of human capital of labor economics based on artificial intelligence, Journal of Intelligent \& Fuzzy Systems (JIFS).

[20] C.Q. Zhang and Y.P. Guan, Research on related technologies of vision target tracking based on discrete differential algorithm for deep learning, Journal of Intelligent \& Fuzzy Systems (JIFS).

[21] Z.Y. Liang, L. Liang, Y.Z. Cai and A. Bertoni, The influence factors of the stability of tailings dam based on multi-source information fusion method, Journal of Intelligent \& Fuzzy Systems (JIFS).

[22] X.G. L, D. L, H.Y. Huang and J.F. Wang, Research on large data intelligent search engine based on multilayer perceptive botnet algorithm, Journal of Intelligent \& Fuzzy Systems (JIFS).

[23] B.X. Ding, W. Chen and Y.Q. Huang, The construction of internet data mining model based on cloud computing, Journal of Intelligent \& Fuzzy Systems (JIFS).

[24] R. Liu, J. Liang and M. Alkhambashi, Research on breakthrough and innovation of UAV mission planning method based on cloud computing-based reinforcement learning algorithm, Journal of Intelligent \& Fuzzy Systems (JIFS).

[25] T.L. Liu, Driving model of innovation of business model based on cloud computing, Journal of Intelligent \& Fuzzy Systems (JIFS).

[26] X.Z. Yin and B. Dylan, Recommendation algorithm based comparative education matching model, Journal of Intelligent \& Fuzzy Systems (JIFS).

[27] X. Li and H. Robin, Construction and analysis of hidden markov model for piano notes recognition algorithm, Journal of Intelligent \& Fuzzy Systems (JIFS).

[28] S.D. Li, J. Xu and S. Cele, Application of graph theory in transportation linkage in logistics management and its computer aided model design, Journal of Intelligent \& Fuzzy Systems (JIFS).

[29] X.J. Zhang and B. Alijla, Research on inheritance and innovation mode of erhu art development based on intelligent algorithm, Journal of Intelligent \& Fuzzy Systems (JIFS).

[30] Y. Bin and D. Mandal, English teaching practice based on artificial intelligence technology, Journal of Intelligent \& Fuzzy Systems (JIFS).

[31] X.X. Ran and M. Hossain, Recommendation algorithm based business english writing training strategy, Journal of Intelligent \& Fuzzy Systems (JIFS).

[32] Z.H. D and Z. Lei, The design of automotive electronic control suspension system based on digital simulation, Journal of Intelligent \& Fuzzy Systems (JIFS).

[33] S.Z. He, Innovative talents training mode of science and engineering universities based on the human resources demand of modern enterprise in IoT technology, Journal of Intelligent \& Fuzzy Systems (JIFS).

[34] Y. Ma, The influence mechanism of financial and monetary policy on economic restructuring based on wireless network technology, Journal of Intelligent \& Fuzzy Systems (JIFS).

[35] Y.K. Li, L. Chu, Y.J. Zhang, C. Guo, Z.C. Fu and J.B. Gao, Intelligent transportation video tracking technology based on computer and image processing technology, Journal of Intelligent \& Fuzzy Systems (JIFS).

[36] M. Zhang, Application and research of automatic password update technology based on the development of wireless network, Journal of Intelligent \& Fuzzy Systems (JIFS).

[37] Q.W. Yuan, The construction mechanism and algorithm of cross border E-commerce export logistics mode from the 
perspective of value chain, Journal of Intelligent \& Fuzzy Systems (JIFS).

[38] J.Y. Huang and G.N. Wong, The function and significance of the history teaching of art design in the development of network of things of business design, Journal of Intelligent \& Fuzzy Systems (JIFS).

[39] W. Wang and D. Mandal, Research on the construction of teaching platform of drama film and television literature based on IoT, Journal of Intelligent \& Fuzzy Systems (JIFS).

[40] D.C. Xu and T.S. Rappaport, Construction on teaching evaluation index system of track and field general course for physical education major in light of wireless network technology, Journal of Intelligent \& Fuzzy Systems (JIFS).

[41] Z.Q. Sang and R.H. Zhang, Branch demarcation algorithm based temperature rise online detection system for complete switchgear, Journal of Intelligent \& Fuzzy Systems (JIFS).

[42] J. Ma, H.H. Yu, D. Wang and C.C.R. Pu, Design and creation of salar acoustical phonetics parameter database, Journal of Intelligent \& Fuzzy Systems (JIFS).

[43] P.Y. Yang, L.C. Chou and Z.A. Wang, Estimation of the labor market information- an empirical study in taiwan, Journal of Intelligent \& Fuzzy Systems (JIFS).

[44] Y.W. Li, Y.T. Yang, S.Q. Ma, L. Li, Y.J. Wang, X.B. Liu and R.H. Xie, Theoretical model construction and structure optimization of electromagnetic flow transducer based on neural network, Journal of Intelligent \& Fuzzy Systems (JIFS).

[45] X.Y. Li, R.Q. Fan, H.L. Zhang, T.L. Li and C.Y. Pang, Twodimensional wavelet synopses with maximum error bound and its application in parallel compression, Journal of Intelligent \& Fuzzy Systems (JIFS).

[46] L.B. Zhou, T. Huang and C.Y. Guan, Real-time detection of cole diseases and insect pests in wireless sensor networks, Journal of Intelligent \& Fuzzy Systems (JIFS).

[47] Z.M. Liu, Y. Xie and H. Zhang, Simulation of passenger behavior and crowd stampede risk on escalator, Journal of Intelligent \& Fuzzy Systems (JIFS).

[48] H.D. Zhang, Fault diagnosis and life prediction of mechanical equipment based on artificial intelligence, Journal of Intelligent \& Fuzzy Systems (JIFS).

[49] G.H. Zhang, P. Brown and G.B. Li, Research on personal intelligent scheduling algorithms in cloud computing based on BP neural network, Journal of Intelligent \& Fuzzy Systems (JIFS).

[50] B. Sun, M. Wei, C.F. Yang and A. Ceder, Solving demandresponsive feeder transit service design with fuzzy travel demand: A collaborative ant colony algorithm approach, Journal of Intelligent \& Fuzzy Systems (JIFS). 